$$
070-17417 \operatorname{Tin} x 63.808
$$

\title{
ELECTRIC FIELD OBSERVATIONS OF IONOSPHERIC WHISTLERS
}

\section{CASE FILE \\ COPY}

N. C. MAYNARD

T. L. AGGSON

J. P. HEPPNER

DECEMBER 1969

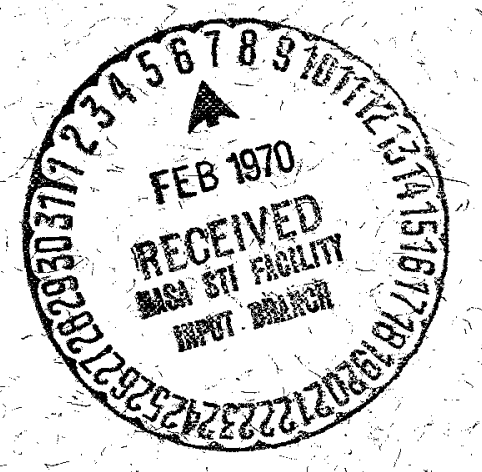

GSFC GODDARD SPACE FLIGHT CENTER

GREENBELT, MARYLAND 


\section{ELECTRIC FIELD OBSERVATIONS}

OF IONOSPHERIC WHISTLERS

N. C. Maynard

T. L. Aggson and

J. P. Heppner

December 1969 
A Nike-Tomahawk sounding rocket was launched near local noon on November 8, 1966 from Wallops Island with a triaxial electric field experiment using long cylindrical dipoles for sensors. Twenty ELF whistlers with dispersion constants ranging from .15 to $.85 \sec ^{\frac{1}{2}}$ were detected and identified as having propagated up to the rocket through the $E$ region from the earth-ionosphere waveguide. The data extend to the smallest dispersion ever encountered and the lowest frequencies observed on ionospheric whistlers $(60 \mathrm{~Hz})$. The waves were eliptically polarized, becoming more nearly circular at higher altitudes. Analysis showed good agreement between the experimental results and quasi-1ongitudinal (QL) propagation theory for amplitude, dispersion, and polarization characteristics of the wave once one is away from the D to E region boundary. No appreciable effects of negative ions or protons were observed in the dispersion of the whistlers. 
INTRODUCTION

A long history exists of VLF and ELF measurements from ground based instruments, (see Helliwe11, 1965). Storey, (1953), postulated that whistlers were created by atmospherics and propagated in the circularly polarized mode roughly along magnetic field lines in space from the northern to southern hemispheres and vice versa. Recently satellite and rocket measurements have added to the data and given a better understanding of VLF emissions and ion effects on the dispersion of waves propagating in the whistler mode. Most previous measurements, including the first by (Cain, et. a1., 1961) have been made in the magnetic mode (e.i., Gurnett, et. a1., 1965; Gurnett, 1966; Smith and Angerami, 1968; He11iwe11, 1969 (review); and the references contained therein). Measurements of the electric field are relatively new and have been confined to single or double axis measurements (Gurnett, et a1., 1969; Shawhan and Gurnett, 1968; Scarf, et. a1., 1968; Maynard and Heppner, 1969; and the references contained therein), or to fixed frequency propagation experiments (Storey and Cerisier, 1968).

The rocket experiment reported here represents the first long dipole 3 axis measurement with wide band telemetry such that the complete wave characteristics are determined. Hence, it is possible to check the experiment against propagation theory (see He11iwe11, 1965) for polarization, dispersion, amplitude and direction of the waves. It is also apropos to study whistlers in the $\mathrm{E}$ region because the angle of injection into the E region of the whistler will determine whether it can be guided along the field lines, and the small amount of dispersion which occurs prior to entry into the ionosphere can be observed. 


\section{THE EXPER IMENT}

The Nike-Tomahawk sounding rocket (NASA 18.04) was launched from Wa11ops Island on November 8, 1966 at 12:32 hours local time. The instrumentation included a three axis electric field experiment using the double floating probe technique (Aggson, 1969) in which the difference in floating potential between the two probes is monitored. The potential difference is the product of the length between the midpoints of the probes and the component of the electric field and $\vec{V} \times \vec{B}$ vectors along the axis of measurement. The differential outputs of each axis were amplified by 100 with capacitively coupled amplifiers (to separate the A.C. components) and used to modulate high frequency, wide-band VCO's for wide-band, real time data. The response (see Figure 1) was rolled off on the low frequency side by the amplifier with the $3 \mathrm{db}$ point at $90 \mathrm{~Hz}$ and on the high frequency side by the response of the ground station (10 $\mathrm{kHz}$ max. in the data presented in this paper).

The cylindrical antennas were 30 feet long with the inner 15 feet insulated from the plasma. This gave a 15 meter baseline between the mid-points of the active elements. The antenna configuration is shown in Figure 2. Thus, the sensitivity was such that $1 \mathrm{mv}$. input (or $67 \mu \mathrm{v} / \mathrm{meter}$ field strength) produced 0.1 volts output from telemetry, and noise levels down to about $10 \mu \mathrm{v} / \mathrm{m}$. could be detected neglecting noise contributions from the payload and ground station. The rocket was despun before deployment, and the spin was further reduced by deployment. The final motion was a slow tumble with a period of about 3 minutes; hence, the spatial orientation of each axis varies slowly in time.

The use of these long dipoles should be contrasted with the short base line experiments of Scarf, et.al., (1966) and Scarf, et. al., (1968). Assuming an index of refraction of the order of 100 , the wave length of 
a $3 \mathrm{kHz}$ wave in the ionosphere is 1000 meters which is two orders of magnitude greater than the dipole antenna base line. The $\mathrm{E}=$ Vmeas./d relationship is thus unambiguous. For electrostatic waves (Scarf, et. al, 1968) this relation is not necessarily true, because electrostatic waves can have wave lengths of the order of or greater than the Debye length which is of the order of centimeters in the ionosphere. For electrostatic waves of these wavelengths, the electric field is given by a frequency dependant relation $E=V_{\text {meas. }} / \lambda$ where $\lambda$ is the wavelength $(\lambda \ll d)$. The only A.C. fields measured, however, were easily recognized as whistler-like disturbances from their dispersion characteristics; thus on $1 y$ the whistler mode need be considered here.

\section{THE RESULTS}

The launch time, chosen to optimise the D.C. objectives of the experiment, was at midday when minimum whistler activity would be expected. During the flight, which lasted approximately five minutes above the $100 \mathrm{~km}$ leve1, no whistlers were observed above $100 \mathrm{~km}$ with dispersion constants long enough to have propagated through the magnetosphere from the southern hemisphere. Thus, all of the twenty observed whistlers had to have come from the local ionosphere. As the dispersion constants of these whistlers ranged from 0.15 to $0.82 \mathrm{sec} \frac{1}{2}$, they are all short fractional hop whistlers coming from the local earth ionosphere waveguide.

The noise levels observed from each of the three axes were in general less than $50 \mu \mathrm{v} / \mathrm{m}$, if this noise is interpreted as having wavelengths greater than 10 meters. If the source of this noise was electrostatic with wavelengths of the order of $10 \mathrm{~cm}$., this upper limit of background electric field strength would correspondingly be less than $5 \mathrm{mv} / \mathrm{m}$. Figure 3 illustrates three examples of background noise taken from both up and down 
legs and from near apogee. Part of this noise is from the commutators and other electronic equipment on board the vehicle.

Figure 4 depicts raw data (signals above $1 \mathrm{KHz}$ attenuated by the chart recorder) for two whistlers. The lower whistler observed at $171.2 \mathrm{~km}$, altitude had a maximum signal amplitude of $450 \mu \mathrm{v} / \mathrm{m}$ resulting in a signal-to-noise ratio of 10 to 1 . The typical whistler observed had a maximum field strength of 100 to $200 \mu \mathrm{v} / \mathrm{m}$. This whistler had the lowest observed frequency components, down to $60 \mathrm{~Hz}$, and was basically eliptically polarized. Having just entered the ionosphere $(111.7 \mathrm{~km})$, the upper whistler had the shortest dispersion of the observed whistlers. One also notes that it was almost completely linear in polarization.

In some cases the rocket was oriented so that one component of the whistler was below the noise leve1. An example of this is shown in Figure 5 in which the raw data from the two remaining axes are plotted against time. No attempt was made to smooth the data. The high degree of circular polarization is evident. The sense is right-handed corresponding to the ordinary wave (see Helliwe11, 1965).

In a number of cases, but not in all, frequencies of several $\mathrm{kHz}$ were observed prior to the ELF portions. The range between 700 to 800 $\mathrm{Hz}$ and $3 \mathrm{kHz}$ was naturally attenuated below the noise level, as explained later. The amplitude of the VLF portion sometimes ranged higher than $800 \mu \mathrm{v} /$ meter with ratios of the maximum VLF to maximum ELF portions being as high as 8 to 1 and typically near 2 to 1 . A spectral contour plot of a whistler observed at $196 \mathrm{~km}$ is shown in Figure 6 to illustrate the observed dead band.

At certain times during the flight modulation from commercial broadcast stations was received on the $\mathrm{AC}$ channels. The stations would fade in and out as the rocket moved through its trajectory. A theory 
by (Kawashima, 1968) explains this as rectification by the plasma at the plasma frequency. Hence as the vehicle passed through a region where the plasma frequency equaled the broadcast frequency, the signa1 would be rectified and the modulation detected by the antennas.

\section{ANALYSIS}

\section{Amplitude}

An averaged amplitude spectrum was obtained using the data from four whistlers recorded between 170 and $211 \mathrm{~km}$. having both ELF and VLF frequency components. To equalize the contributions each whistler was normalized in amplitude to unity at the ELF maximum. The resulting spectrum is shown in Figure 7. This spectrum has also been corrected for the amplifier response curve of Figure 1 . One notes the ELF maximum to be at $300 \mathrm{~Hz}$ with the amplitude gradually falling off at lower frequencies. The aforementioned dead band is seen with the VLF maximum being above $5 \mathrm{kHz}$.

The shape of the spectrum is determined by the source, attenuation from propagation in the earth-ionosphere waveguide and attenuation from propagation in the ionosphere. It has been shown theoretically and by ground based measurements (Alpert, et. a1., 1966) that the dead band between 800 and $3000 \mathrm{~Hz}$ is the result of propagation over large distances through the earth-ionosphere cavity before entering the ionosphere. This is due to attenuation in the waveguide where the cavity spacing is close to one half wavelength (Helliwe11, 1965). Curves for the spectrum of the electric field after having propagated various distances from a constant intensity source are given by (Alpert, et. al., 1966).

Ful1 wave calculations have been made by (Aksinov and Lishin, 1967) for the propagation losses through the lower ionosphere, considering a multicomponent plasma between 50 and $200 \mathrm{~km}$, a vertically incident wave 
and a vertical magnetic field. For comparison here, their resulting transmitted energy ratio was converted to a relative electric field ratio by taking its square root and multiplying by the fourth root of the frequency, to correct for the change in index of refraction. By taking the product of this ratio with the curve obtained by (Alpert, et a1., 1966), for a propagation distance of $1000 \mathrm{~km}$, the solid curve in Figure 6 was derived. $\operatorname{Had} 2000 \mathrm{~km}$ been used, the maximum in the ELF region would have broadened and shifted to below $200 \mathrm{~Hz}$ and the dead band would have been wider.

Considering the many approximations involved including a constant amplitude source, vertical incidence on the ionosphere and vertical magnetic field, the agreement with the data is good. From the agreement of the data and calculation, it is probable that the whistlers traveled over $1000 \mathrm{~km}$ before entering the ionosphere. In support of this, there was no thunderstorm activity near Wallops Island that day. It is noted that the large ratio of VLF and ELF amplitude in the calculated curve was seen in some cases although it was not typical.

\section{Dispersion}

Since the frequencies observed here are well below the nose frequency the dispersion of the whistler can be described by the relations

$$
D=T \sqrt{f}
$$

where $T$ is the group delay, $f$ the frequency, and $D$ is the dispersion constant. Hence, the dispersion constant can be shown to be the slope of the T vs. $f^{-\frac{1}{2}}$ plot of the measured wave. $T$ may also be related to the parameters of the medium in the Storey or quasi longitudinal ( $Q$ L) approximation of Appleton Hartree theory by

$$
T=\frac{1}{2 c} \int_{\text {PATH }} \frac{f_{0}}{\sqrt{f\left(f_{i+} \cos \theta\right)}} d s
$$


where $f_{0}$ is the electron plasma frequency, $f_{H}$ is the electron gyro-frequency, and $\theta$ is the angle between the wave normal and the magnetic field. Thus in the low frequency approximation

$$
D=\frac{1}{2 C} \int_{\text {rath }} \frac{f_{i}}{\sqrt{f_{i+} \cos \theta}} d S
$$

This includes only dispersion from electrons and doew not include ion effects (see Storey, 1953 and He11iwe11, 1965).

Upon entering the lower edge of the ionosphere the index of refraction in the ELF range increases rapidly by two orders of magnitude. by Snel1's 1aw, a wave intersecting this lower boundary will be refracted nearly vertical, hence the wave normal can be assumed to be in the vertical direction for all the whistlers above the lower E region. This fixes the angle $\theta$ to be the magnetic dip angle which is $20.5^{\circ}$ at Wallops Island. The ray path or path of energy flow needed for integration of Equation 3 will not necessarily follow the wave normal, but as shown in the appendix the ray path direction is to a good approximation, at an angle from the wave normal whose tangent is $1 / 2$ of $\tan \theta$. The integration in Equation 3 was carried out numerically starting at $100 \mathrm{~km}$ using an inverse cube fall-off of magnetic field and the electron density as measured by the Wallops Island ionosonde. Since the slope of $\mathrm{N}_{e}$ vs $\mathrm{h}$ was monotonically increasing, the resulting values of $\mathrm{N}_{e}$ are unambiguous; however, small errors in the altitude assigned are inherent in the measurement. A second calculation was done as a check using the electron density measured on board the vehicle by the VLF Doppler shift technique of(Maeda, et al., 1964). The results are plotted as the solid and dashed lines respectively in Figure 7.

Since whe whistlers were too short in duration to get an accurate measurement of the dispersion from a sonogram type of analysis, they were displayed on strip charts and the period of each half cycle of the wave was 
measured. The time corresponding to this period was assumed to be the center time of the half cycle. Zero crossings of the wave were used as the limits of the measurement interval. The data for each whistler observed were plotted in the form $1 / \sqrt{f}$ versus $t$. Three examples of these curves taken from different attitudes are shown in Figure 8. The slope of the curve was then found using a linear least squares fit; the reciprical of this slope is the dispersion constant.

A systematic error is introduced in the lower E region whistlers by the fact that the frequency and amplitude are changing within a half-cycle of the wave, hence the assigned time is not the correct time for the measured period. Analysis showed that this effect resulted in an error of the order of $10 \%$ or less on the whistler with a dispersion consistant of $0.15 \mathrm{sec} \frac{1}{2}$ and correspondingly smaller errors on those with larger dispersion constants. The effect is to increase the dispersion constant. The resulting value of the dispersion of each whistler is plotted in Figure 9. The error bars are a $3 \sigma$ variation taken by using the difference between the slope of the line between each point and the initial time and the least squares slope and do not include the systematic error. The agreement with the above calculated curve is good down through $130 \mathrm{~km}$ and deviates slightly below that. This can be accounted for in that:

(a) there is some dispersion at low frequencies in the earth-ionosphere waveguide and in the $\mathrm{D}$ region, (b) that the approximations used in the above calculations breakdown within a few wavelengths of a sharp boundary, (c) and that the systematic error discussed in the previous paragraph exists. For eight of the whistlers the VLF portions were analyzed and the corresponding dispersion constants were found. The ELF and VLF results for these eight whistlers are summarized in Table 1 . Variations in the dispersions can be expected from the higher frequency of the VLF portim (resulting in larger errors in the low frequency approximations) and from the 
effect of protons. (Barrington and Nishizaki, 1960) approximated these effects by the relation.

$$
\begin{aligned}
& t f^{\frac{1}{2}}=\frac{1}{2 c} \int_{\text {AATH }} \frac{f_{0}}{f_{H}^{1 / 2}}\left\{\frac{1}{\left(1-\frac{f}{f_{H}}\right)^{\frac{3}{2}}}+\frac{1-\epsilon\left[f_{i+} /\left(f_{i H}+f\right)\right]^{2}}{\left(1-\frac{\epsilon f_{i H}}{f_{i+H}+f}\right)^{\frac{1}{2}}}-1\right\} d s \\
& \text { where } f_{H} \text { is the electron gyro frequency } \\
& \text { fiz is the proton gyro frequency } \\
& \epsilon \text { is the ratio of the number of } \\
& \text { hydrogen ion to electrons. }
\end{aligned}
$$

The net result is that a slightly larger dispersion constant would be expected at VLF frequencies compared to the ELF frequencies.

A1though the data are scattered, the average ratio of VLF to ELF dispersion was found to be 1.01 with a $3 \sigma$ value of .06 . If we assume a constant magnetic field and that $\epsilon$ is an average value over the integration region, then the parenthetical factor can be removed from the integral. The expected ratio was then calculated for an $\varepsilon$ of zero and found to be 1.005 . The effect of the protons would be to slightly increase the ratio. (Brinton, et.a1., 1969), have found the number of protons at $250 \mathrm{~km}$. to be $2 \times 10^{2} / \mathrm{cm}^{3}$ and falling off rapidly below that altitude. Considering the accuracy of the measurement, and that $n$ is of the order of $10^{5} / \mathrm{cm}^{3} \epsilon$ must be assumed to be effectively zero.

The possibility of a strong interaction of whistlers with negative ions in the lower ionosphere was discussed by Shawhan, (1966). Due to the resonance of the rotation of circularly polarized whistlers with the circular rotation of negative ions at their gyrofrequencies, Shawhan showed that the Appleton Hartree dielectric constant diverges at a frequency near the negative ion gyrofrequency, if negative ions are present in the lower ionosphere. Shawhan chose a rather speculative ion Hē for his calculations; the existance of this ion has not been detected in the lower ionosphere. However, negative ion mass spectrograph experiments have detected $0^{-}$ions in low quantities in the lower $\mathrm{E}$ region (Narcisi, et. al., 1969) and (Johnson and Heppner, 1956), and it would be of considerable 
interest to repeat Shawhan's calculations for that ion. It is somewhat surprising that these ions do not seem to produce a measurable effect below $100 \mathrm{~Hz}$ (see Figure 8); however, the response of the amplifier prevents looking directly at the $0^{-\infty}$ gyrofrequency.

\section{Polarization}

The polarization of all the whistlers except those in the lower E region was eliptical and in some cases nearly circular. Figure 5 is illustrative of the whistlers observed near apogee. Since the whistlers may have a component in the wave normal direction and magnetic aspect only orients the vehicle on a cone, definition of the wave normal direction and ray path becomes complicated.

The polarization equation is given by (Budden, 1961) as:

$$
\rho=\frac{E_{X}}{E_{Y}}=\frac{i Y_{T}^{2}}{2 Y_{L}(1-X-i Z)} \pm i\left\{\frac{Y_{T}^{4}}{4 Y_{L}^{2}(1-X-i Z)^{2}}+1\right\}^{\frac{1}{2}}
$$

where the $z$ direction is that of the wave normal and

$$
\begin{array}{ll}
\mathrm{Y}=\frac{f_{H}}{\mathrm{f}} & \mathrm{Y}_{\mathrm{t}}=\mathrm{Y} \sin \theta ; \mathrm{Y}_{\mathrm{L}}=\mathrm{Y} \cos \theta \\
\mathrm{X}=\frac{\mathrm{f}_{\mathrm{O}}^{2}}{\mathrm{f}} & \theta=\text { angle of } \mathrm{B} \text { from } \mathrm{z} \text { axis } \\
\mathrm{Z}=\frac{\nu}{\mathrm{t}} & \nu=\text { collision frequency }
\end{array}
$$

As $X>>Y>Z$ for the observed frequencies and ionospheric conditions existing in the $\mathrm{E}$ region and above the expression reduces to

$$
\rho= \pm i
$$

or pure circular polarization. There can also exist a component of the electric field in the wave normal or $z$ direction given by

$$
\frac{E_{Z}}{E_{Y}}=\frac{-i Y_{T}\left(n^{2}-i\right)}{(1-x-i z)}
$$

where $\mathrm{n}$ is the index of refraction. Considering the parameters at $210 \mathrm{~km}$ the index of refraction was calculated to be 373 for a $300 \mathrm{~Hz}$ wave. Correspondingly, an Ez/Ey ratio of about 1.2 would be expected from equation 7 . 
The data from two whistlers where one component of the wave was zero were plotted to obtain the polarization elipse. The elipse was rotated about its minor axis into a circle. The normal to this circle was assumed to be the wave normal and was then compared with possible positions of the local vertical (as determined by the magnetic aspect) in Figure 10. The noted coordinates are those of the vehicle. The agreement is within the accuracy of the measurement. The ratio of the component along the normal to thatperpendicular was in Figure 10a, 1.3, and in Figure 10b, 0.7. It is noted that the index of refraction on which the 1.2 estimate was made is strongly dependent of frequency and that, as one can see from Figure 4, the polarization plot is not precise; hence, the agreement is within expected variations.

\section{CONCLUS IONS:}

The agreement between quasi-longitudinal (QL) propagation theory and experiment for the amplitude, polarization and dispersion characteristics of waves propagating in the whistler mode has been demonstrated in the lower ionosphere. Whereas the simple approximations become crude near the $\mathrm{D}$ to $\mathrm{E}$ region boundary due to the rapidly changing environment, the agreement in the upper $\mathrm{E}$ region and lower $\mathrm{F}$ regions is good. The data extends down to the smallest dispersions ever encountered $\left(0.15 \sec \frac{1}{2}\right)$ and the lowest frequencies observed on atmospheric whistlers $(60 \mathrm{~Hz})$. The agreement of the wave normal data and the dispersion constant data with theory provides a check on the ray path behavior in the lower ionosphere. No appreciable affects of negative ions was observed in the dispersion of the whistlers down to $60 \mathrm{~Hz}$; although changes due to $0^{-}$ might be expected from the work of Shawhan, (1966).

The close experimental-theoretical agreement is an indication that the experiment can be used to accurately monitor electric fields through ELF frequencies. 


\section{ACKNOWLEDGEMENT}

The authors wish to thank Dr. A. Egeland for his work in the reduction of the Doppler shift electron density data used in the theoretical dispersion calculation and for helpful comments. We also thank Miss L. Wescott of ESSA for the reduction of the ionosonde data. Figure 6 was made through the courtesy of Hydrospace Research Corporation. 
Calculation of the ray path angle.

Let $\theta=$ angle of the wave normal with respect to $\vec{B}$.

$\alpha=$ angle of the ray path with respect to the wave normal.

Then from Helliwe11, 1965, p. 34

$$
\tan \alpha=\frac{1}{\mu} \frac{\partial \mu}{\partial \sigma}
$$

where $\mu$ is the index of refraction given by Helliwe11 on p. 29

for the Q.L. approximation as:

$$
\mu^{2}=1+\frac{f_{O}^{2}}{f(f H \cos \theta-f)}
$$

and where $f_{0}=$ the plasma frequency

$f_{H}=$ the electron gyrofrequency

For ELF frequencies in the $E$ and $F$ regions where $f \ll f H$ and

$f \ll f_{o}$, equation A2 may be approximated by:

$$
\mu^{2}=\frac{f_{Q}^{2}}{\mathrm{f} f \mathrm{H} \cos \theta}
$$

Differentiating this with respect to $\theta$ yields:

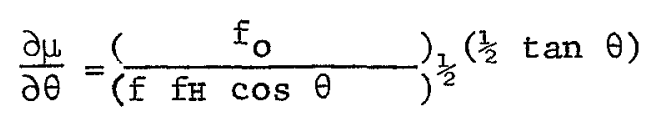

Thus combining A4 with A1:

$$
\tan \alpha=\frac{1}{2} \tan \theta
$$


Aggson, T. L. (1969), Probe measurements of electric fields in space, Atmospheric Emissions, ed. B. M. McCornac and A. Omholt, Van Norstrand Reinhold Co., New York.

Aksinov, V. I. and I. V. Lishin (1967), Influence of ions on the passage of low frequency electromagnetic waves through the ionosphere, translation by A. L. Brichant from Radiotekhnika i Elektronika, 12,703 .

A1'pert, Ja. L., D. S. F1izel and G. A. Michailova (1967), The propagation of atmospherics in the earth-ionosphere waveguide, J.Atm. Terr. Phys., 29, 29.

Barrington, R. E. and T. Nishizaki (1960), Whistler dispersion and exospheric hydrogen ions, J. Geophys. Res., 65, 2581.

Brinton, H. C., M. W. Pharo, III, H. G. Mayr and H. A. Taylor, Jr. (1968), A direct measurement of ion composition and concentration in the daytime $F_{Z}$-region, Goddard Space Flight Center Report, X-621-68-329.

Budden, K. G. (1961), Radio Waves in the Ionosphere, Cambridge University Press, Cambridge, England.

Cain, J. C., I. R. Shapiro, J. D. Stolarik and J. P. Heppner (1961), A note on whistlers observed above the ionosphere, J. Geophys. Res., 66, 2677.

Gurnett, D. A. (1966), A sate11ite study of VLF hiss, J. Geophys. Res., 71 5599.

Gurnett, D. A., S. D. Shawhan, N. M. Brice and R. L. Smith (1965), Ioncyclotron whistlers, J. Geophys. Res., 70, 1665.

Gurnett, D. A., G. W. Pfeiffer, R. R. Anderson, S. R. Mosier and D. P. Cauffman (1969), Initial observations of VLF electric and magnetic fields with the INJUN-5 satellite, J. Geophys. Res., 74, 4631.

He11iwe11, R. A. (1969), Low-frequency waves in the magnetosphere, Magnetospheric Physics ed. by D. J. Williams and G. D. Mead, American Geophysical Union, Washington.

He1liwe11, R. A. (1965), Whistlers and Related Ionospheric Phenomena, Stanford University Press, Stanford, California.

Johnson, C. Y. and J. P. Heppner (1956), Daytime measurement of positive and negative composition to $131 \mathrm{~km}$. by rocket-borne spectrometer, J. Geophys. Res., 61,575 .

Kawashima, Nobuki (1968), Demodulation of amplitude modulated RF waves in a plasma at resonance, Radio Science, 3 (new series), 377.

Maeda, K-I., T. Obayashi, I. Kimura, H. Oya, and T. Ogawa (1964), Rocket observation of the ionosphere by means of the doppler technique, Rep. Ionos. and Space Res., Japan, 18, 329. 
Maynard, N. C., and J. P. Heppner (1969), Variations in electric fields from polar orbiting satellites, Particles and Fields in the Magnetosphere, ed. by B. M. McCormac, D. Reidel Publishing Co., (inprint).

Narcisi, R. S., A. D. Bailey, L. E. Della Lucca, and C. Sherman (1969), Measurement of negative ions in the lower ionosphere (abstract), EOS - Trans. Am. Geophys. Un., 50, 654.

Scarf, F. L., G. M. Crook, and R. W. Fredericks (1966), Survey of VLF electric fields in the magnetosphere with polar obriting spacecraft, 1964-45A, Radio Sci., 1 (new series), 939.

Scarf, F. L., R. W. Fredricks, and G. M. Crook (1968), Detection of electromagnetic and electrostatic waves on OV3-3, J. Geophys. Res., 73, 1723.

Shawhan, S. D., (1966), Negative ion detection in the ionosphere from effects on ELF waves, J. Geophys. Res., 71, 5585.

Shawhan, D. S. and D. A. Gurnett (1968), VLF electric and magnetic fields observed with the Javelin 9.45 sounding rocket, J. Geophys. Res., $\underline{73}, 5649$.

Smith, R. L. and J. J. Angerami (1968), Magnetospheric properties deduced from OGO-1 observations of directed and non-directed whistlers, J. Geophys. Res., 73, 1 .

Storey, L.R.O., (1953), An investigation of whistling atmospherics, Phil. Trans. Roy. Soc. London, Ser. A., 246, 113.

Storey, I.R.O. and J. C. Cerisier (1968), Une interprétation des bandes der bruit an voisinage de la fréquence hybride basse observées an moyen le satellites artificiels, Compt. Rend. Acad. Sci., Paris, $\underline{266}, 525$. 
FIGURE CAPTIONS

Figure 1: Frequency response of the broad band A.C. amplifier used on each axis.

Figure 2: Configuration of the antennas for the triaxial electric field monitor, flight 18.04.

Figure 3: Background noise levels from three different times during the flight.

Figure 4: The ELF portions of two whistlers. The upper whistler has the shortest observed dispersion constant while the lower whistler is typical of those observed at higher altitudes.

Figure 5: Two components of a whistler plotted against time (the third component was zero) illustrating the eliptical polarization. The two dimensional plot is a compressed view of the $312-227 \mathrm{~Hz}$ portion of the three dimensional plot.

Figure 6: A spectral contour plot of a whistler observed at $196 \mathrm{~km}$. illustrating the characteristic dead band between 1 and $3 \mathrm{kHz}$.

Figure 7: Average amplitude spectrum normalized to the ELF maximum compared with a theoretically predicted spectrum (see text).

Figure 8: Three plots of $\sqrt{\mathrm{T}}$ vs. elapsed time from which the dispersion constant was calculated. One of the shortest whistlers, the whistler with the lowest frequency components (also shown in Figure 4) and a whistler near apogee are shown.

Figure 9: The dispersion constant variation with altitude compared with the calculated variation from Appleton-Hartree theory. The solid line represents the calculated value using the ionosonde data while the dashed line is that using the Doppler shift data (see text).

Figure 10: A polar plot in rocket coordinates showning the locus of the normal to circular $\overrightarrow{\mathrm{E}}$ (should be nearly vertical from Snell's Law) compared with the locus of possible local vertical positions derrived from the knowledge of $\vec{B}$ for two whistlers near apogee. 
TABLE 1

Comparisons of ELF and VLF Whistler Dispersions

Altitude o
Observation
$171.2 \mathrm{~km}$.
178.7
183.2
196.3
197.9
206.6
211.4
211.8

$\mathrm{D}_{\mathrm{ELF}}$

$.426 \mathrm{sec}^{\frac{1}{2}}$

.503

.550

.649

.686

.743

.821

.783
$\mathrm{D}_{\mathrm{VLF}}$

$.581 \mathrm{sec}^{\frac{1}{2}}$

.515

.446

.623

.700

.817

.656

.790 


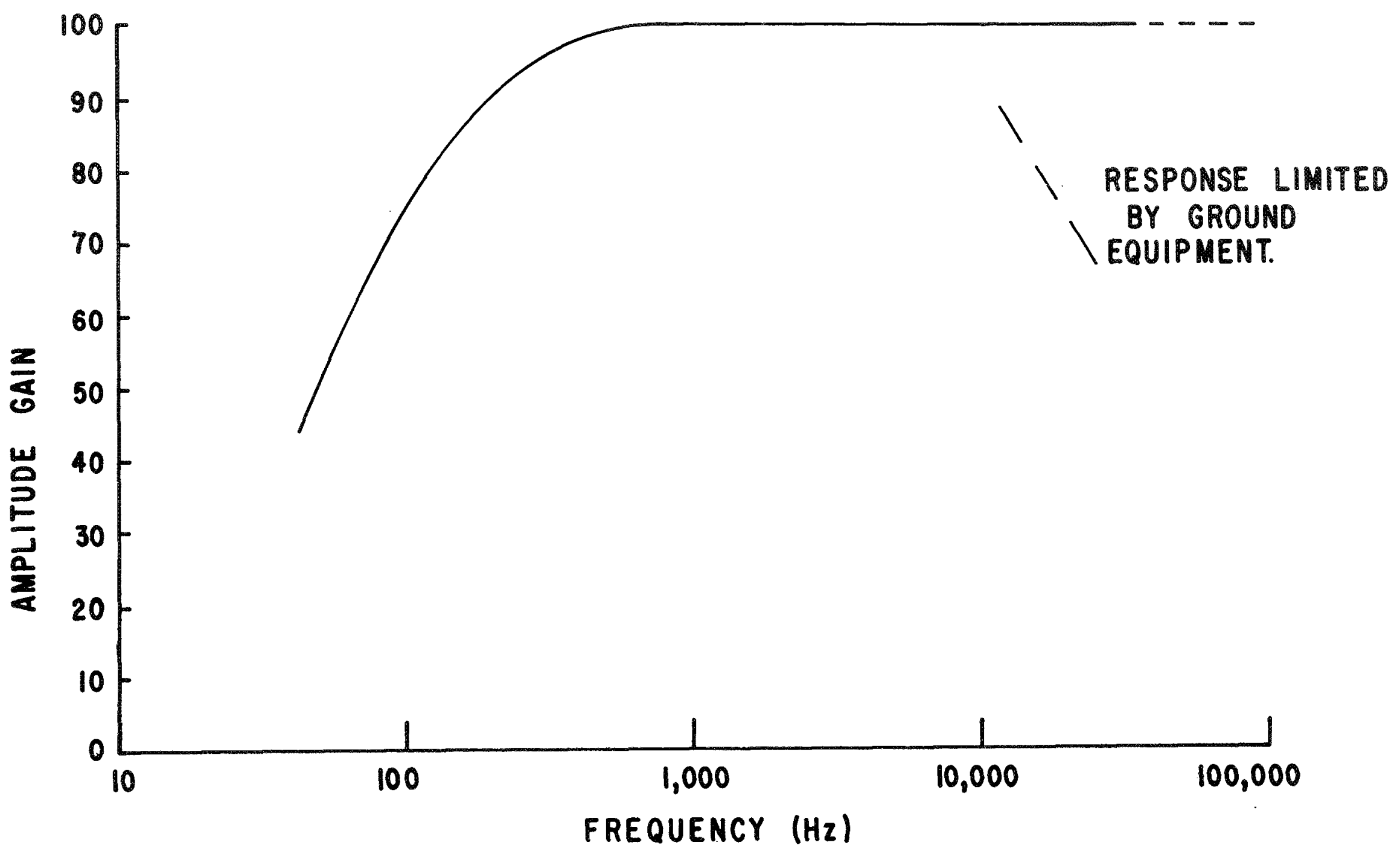




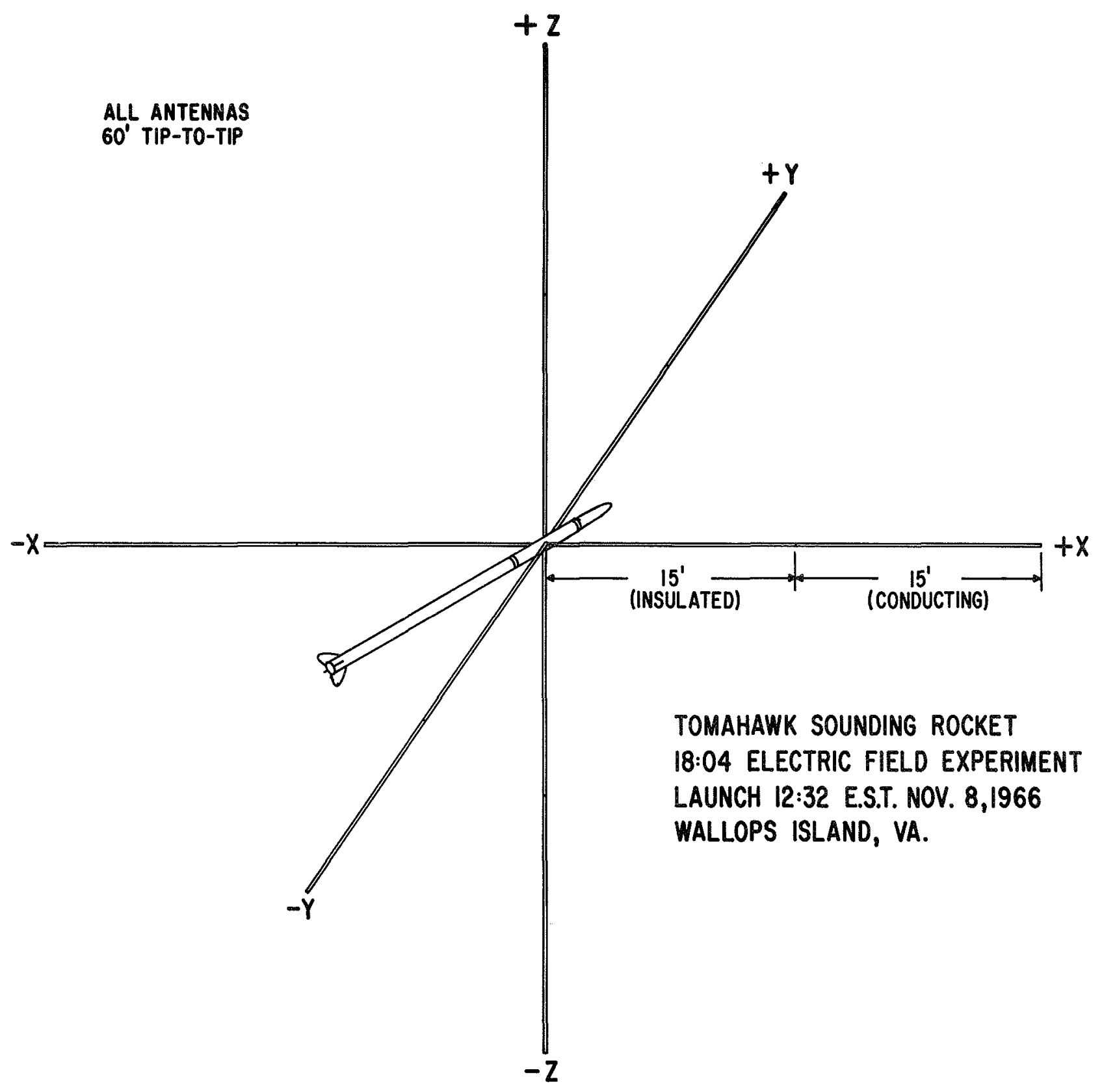

FIGURE 2 
$179 \mathrm{KM}$ UP LEG

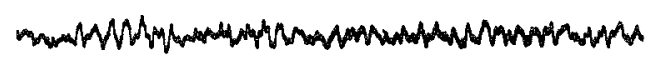

212 KM NEAR APOGEE

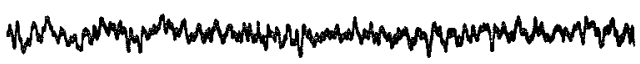

150 KM DOWN LEG

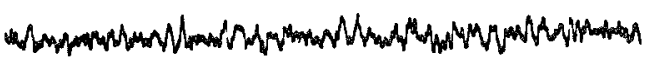

(n)

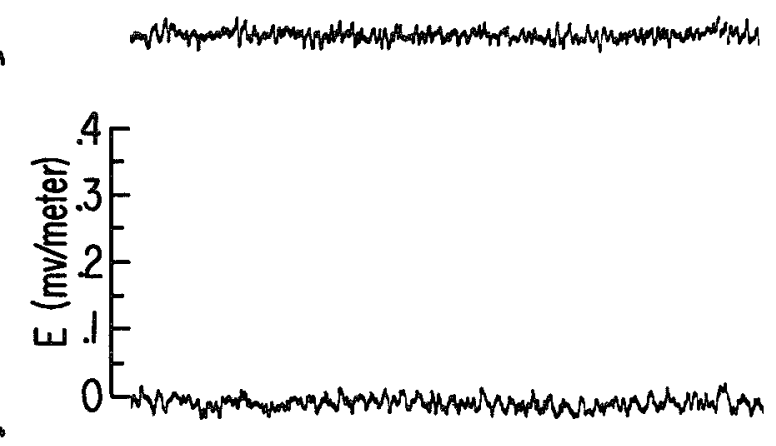
.05

10

$\begin{array}{ll}1 & 1 \\ 0 & .0\end{array}$

$\begin{array}{ccccc}1 & 1 & 1 & 1 & 1 \\ .01 & .02 & .03 & .04 & .05\end{array}$ $\begin{array}{cccc}1 & 1 & 1 & 1 \\ .02 & .03 & .04 & .05\end{array}$

$\begin{array}{lllr}1 & 1 & 1 & 1 \\ 0 & .01 & .02 & .03 \\ \text { TIME } & \text { (sec.) }\end{array}$ TIME (sec.)

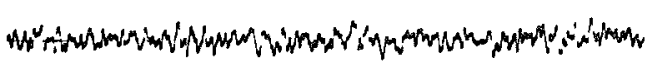

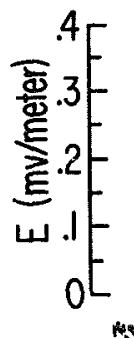

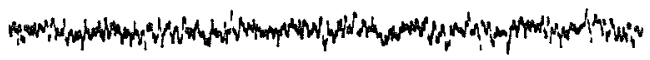

$\begin{array}{ccccccc}1 & 1 & 1 & 1 & 1 & 1 & 1 \\ 05 & 0 & .01 & .02 & .03 & .04 & .05\end{array}$



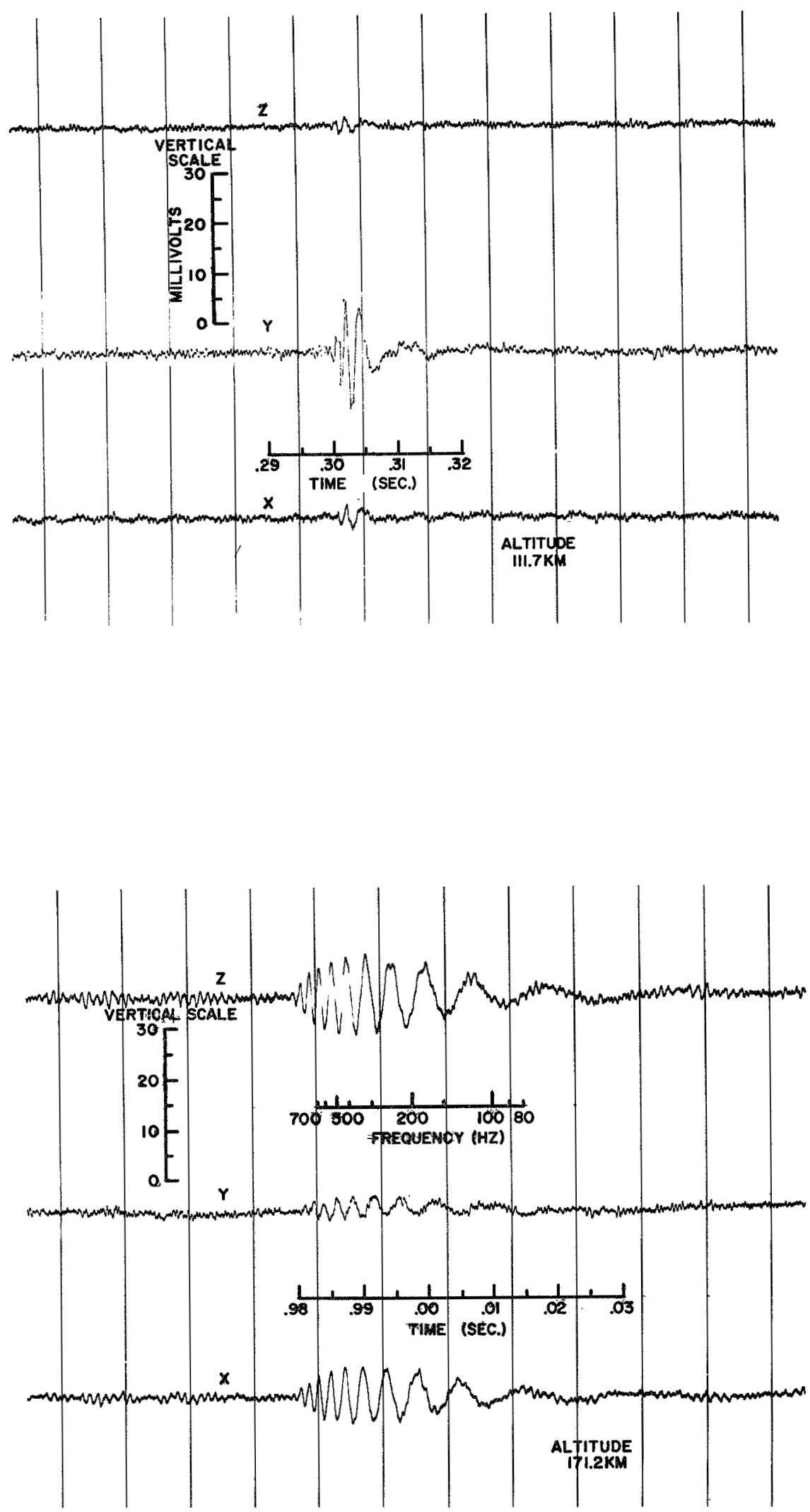


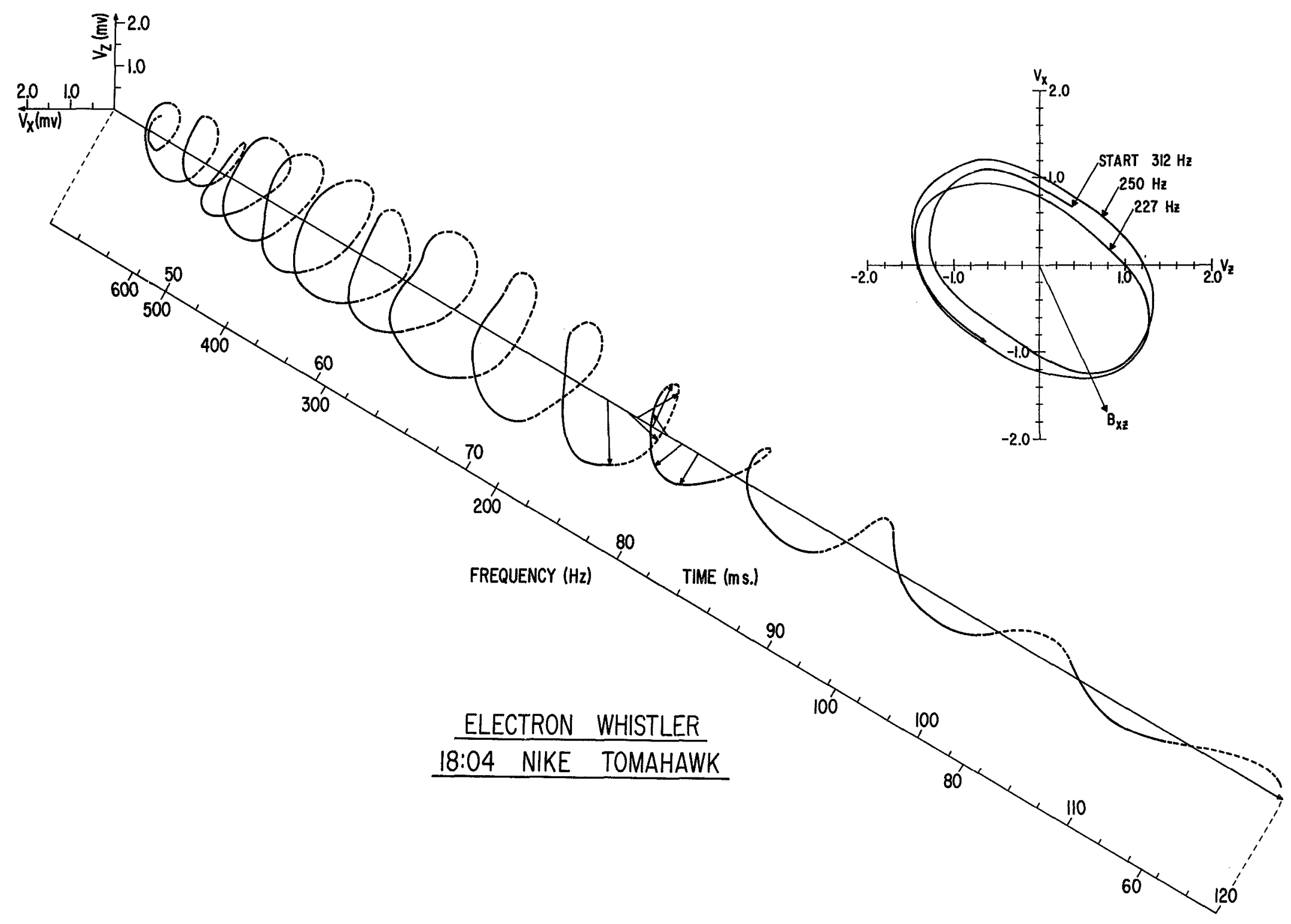

FIGURE 5 


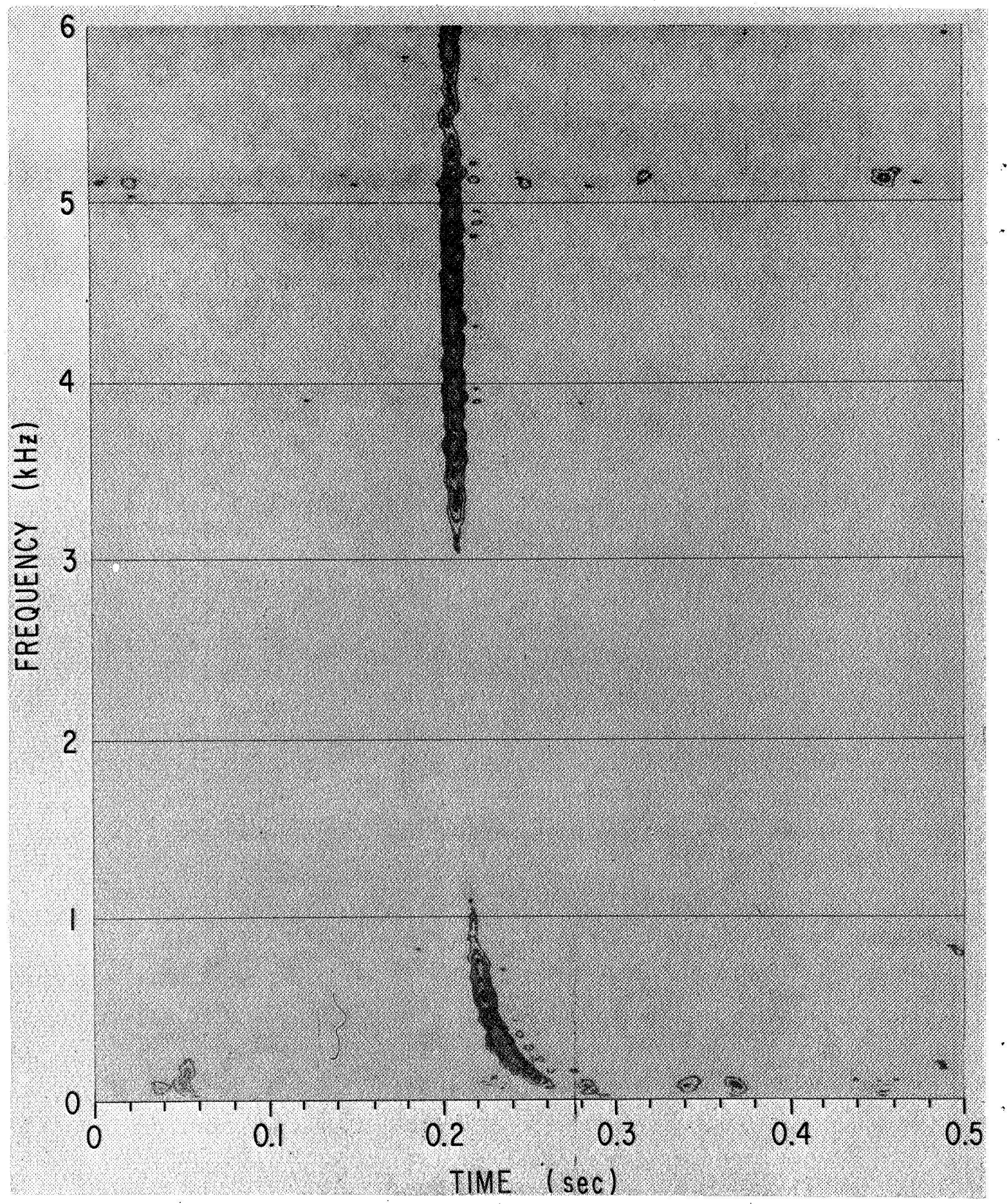

FIGURE 6 


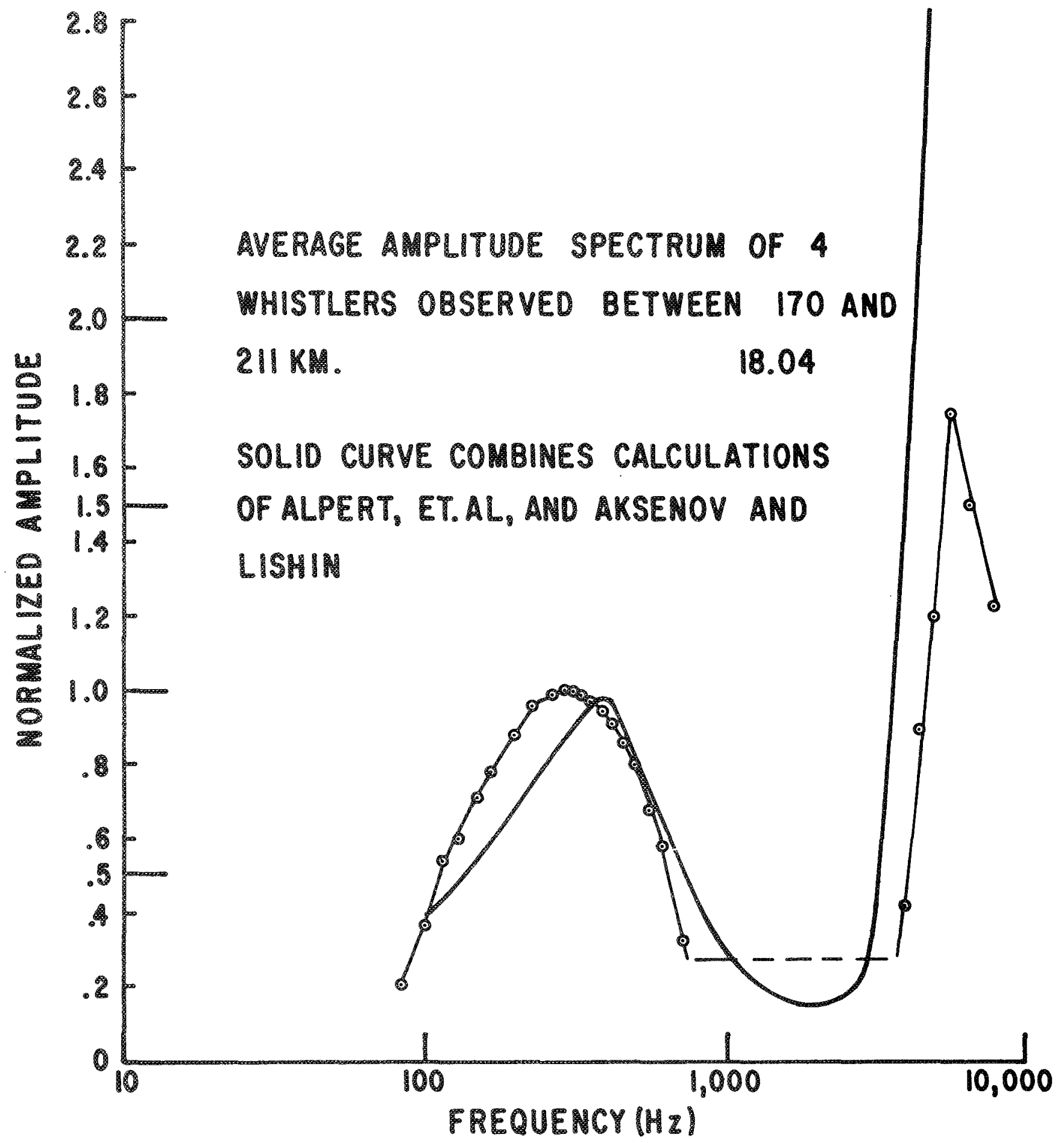

FIGURE 7 


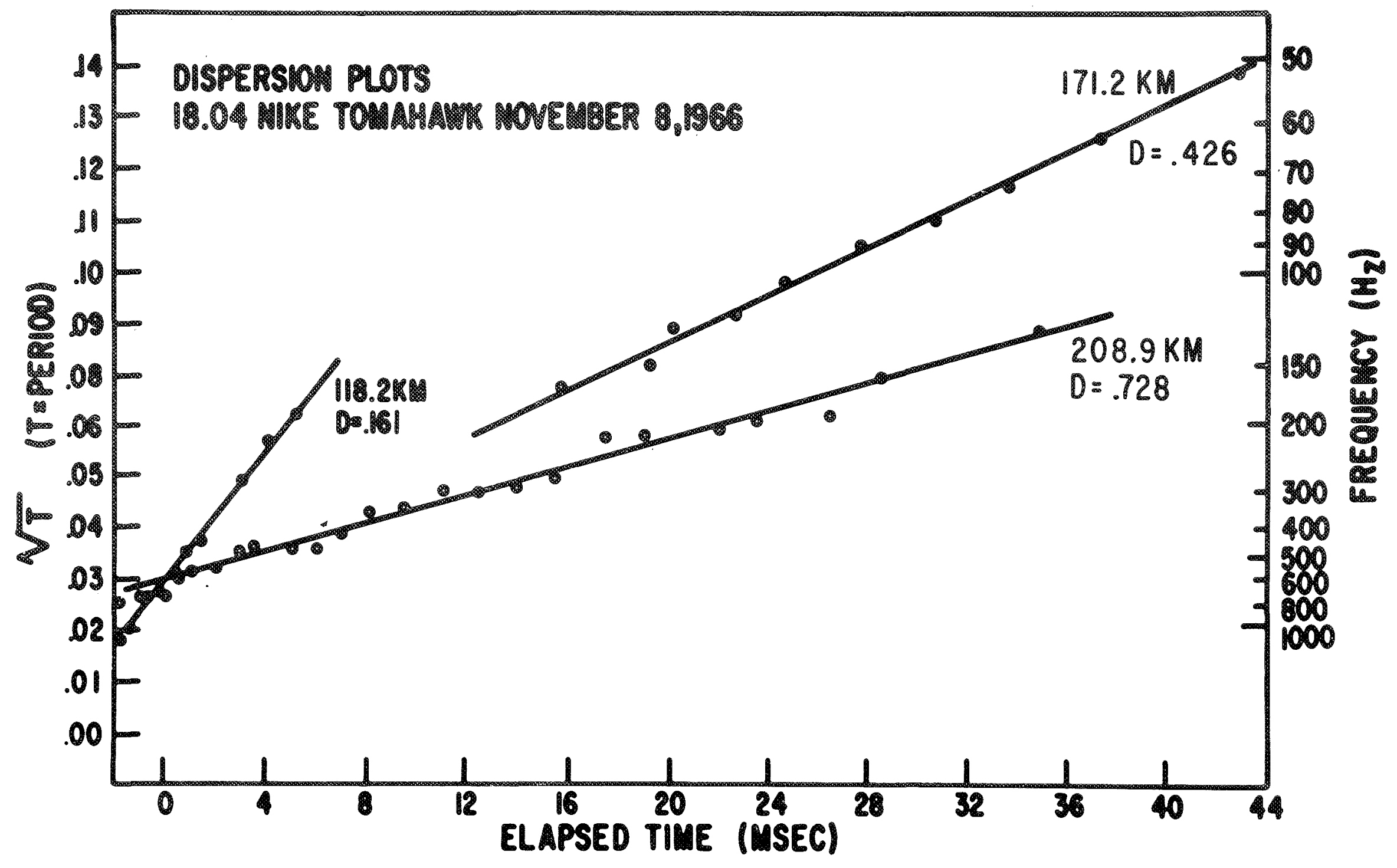

FIGURE 8 


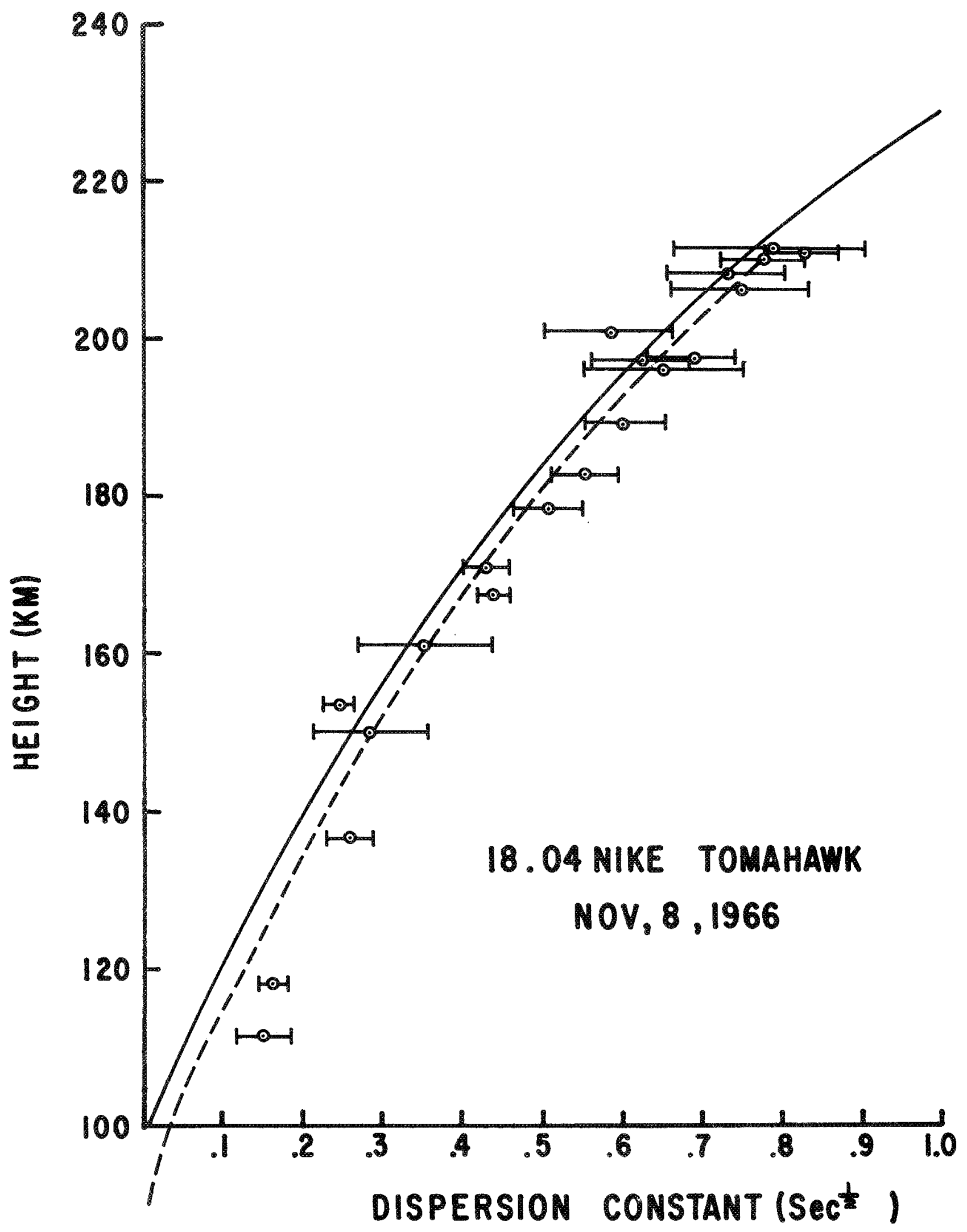

FIGURE 9 


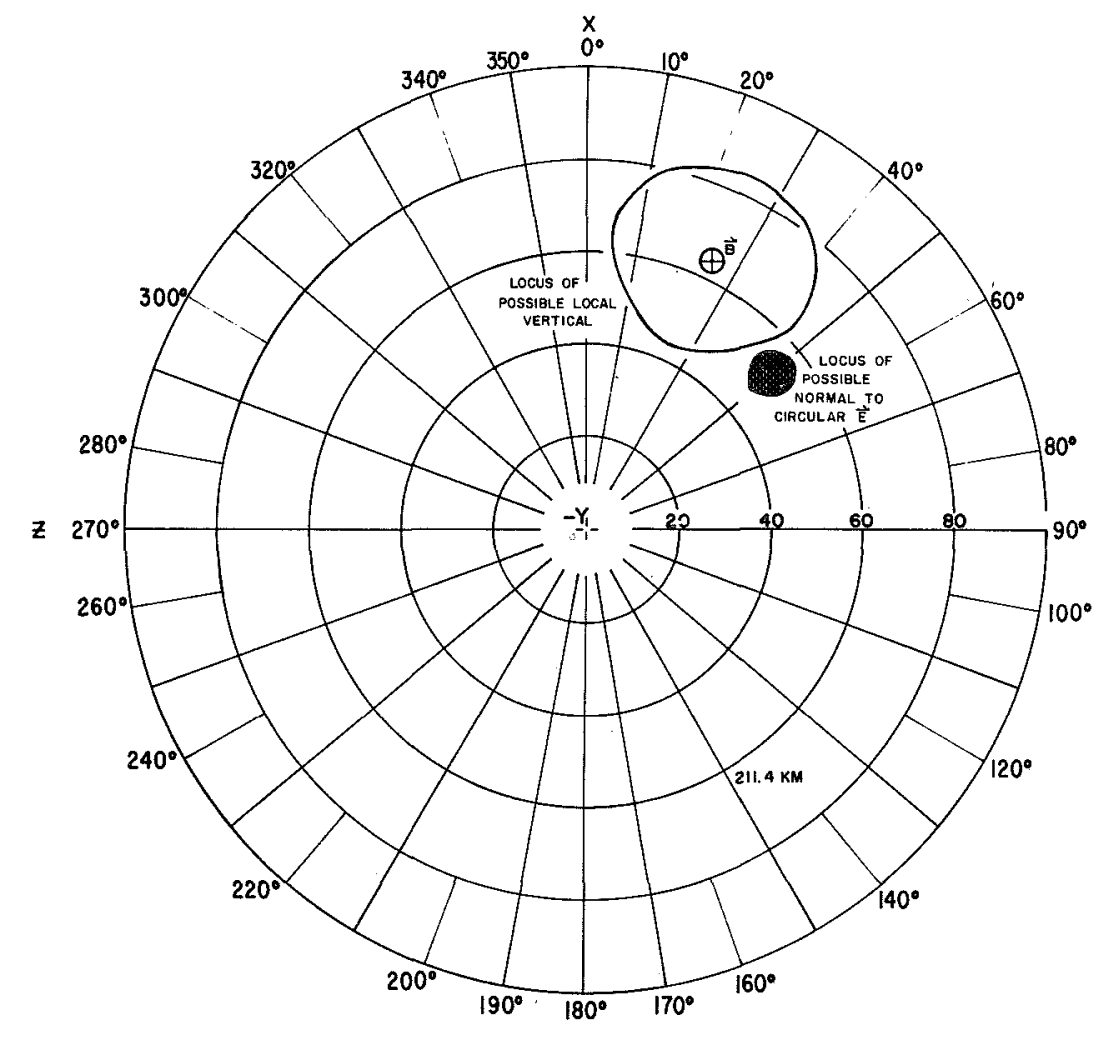

A

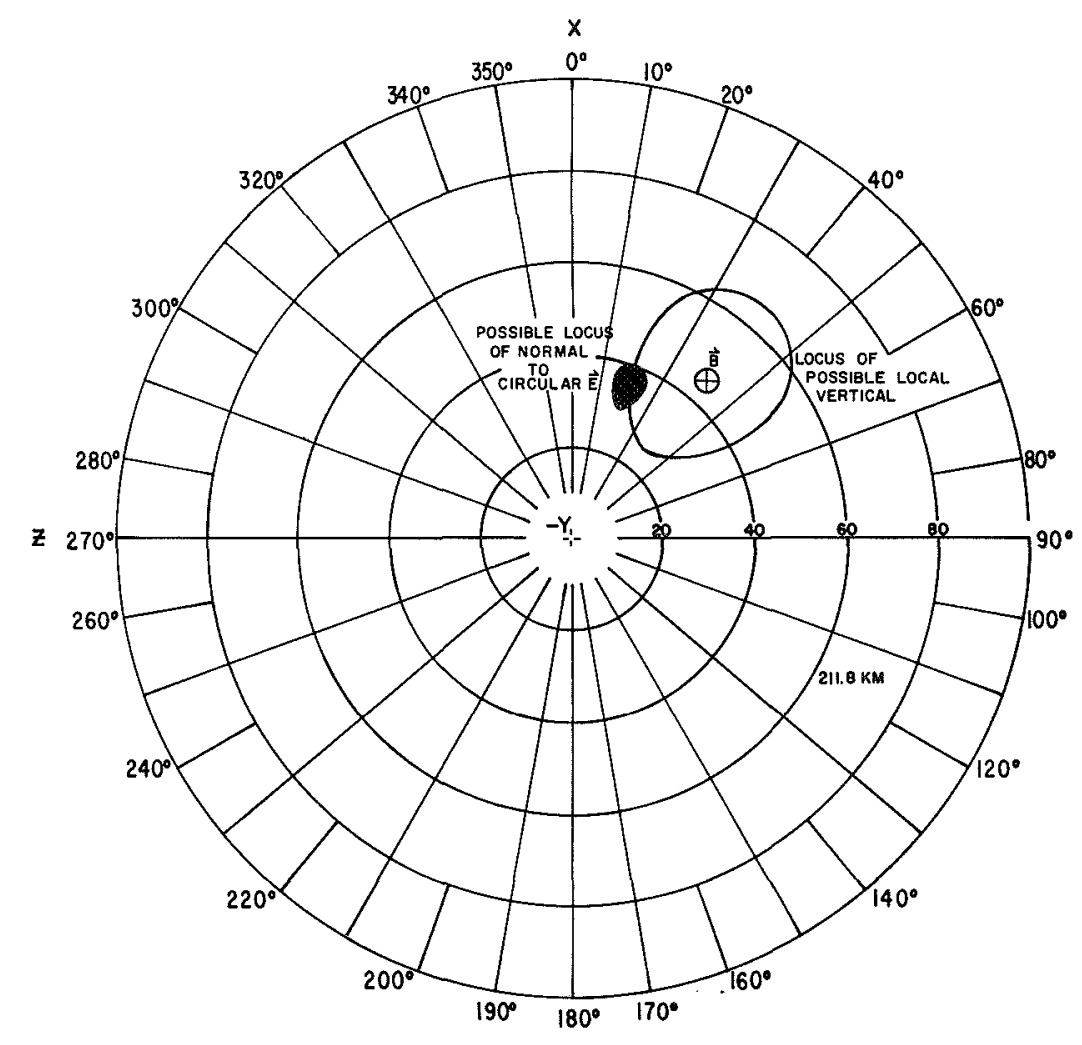

B

FIGURE 10 